\title{
Novel amyloid-forming protein in Escherichia coli
}

\author{
M. Belousov ${ }^{1 *}$, S. Bondarev ${ }^{1}$, A. Kosolapova ${ }^{1,2}$, K. Antonets ${ }^{1,2}$, M. Belousova ${ }^{2}$, \\ A. Sulatskaya ${ }^{3}$, M. Sulatsky ${ }^{3}$, G. Zhouravleva ${ }^{1}$, I. Kuznetsova ${ }^{3}$, K. Turoverov ${ }^{3,4}$, \\ A. Nizhnikov ${ }^{1,2}$ \\ ${ }^{1}$ Saint Petersburg University, St. Petersburg, Russia \\ ${ }^{2}$ All-Russia Research Institute for Agricultural Microbiology, Pushkin, St. Petersburg, Russia \\ ${ }^{3}$ Institute of Cytology RAS, St. Petersburg, Russia \\ ${ }^{4}$ Peter the Great St. Petersburg Polytechnic University, St. Petersburg, Russia \\ *e-mail: belousovmix@gmail.com
}

Key words: secreted metalloprotease, functional amyloids, bacteria, Escherichia coli

Motivation and Aim: Amyloids are protein fibrils with a characteristic spatial structure. In previous studies, using a method for the proteomic screening and identification of amyloids (PSIA) [1], we identified 61 proteins of Escherichia coli that formed detergentresistant aggregates in vivo and without overproduction [2]. Among these proteins, YghJ was the most enriched with bioinformatically predicted amyloidogenic regions. YghJ is a lipoprotein and important virulence factor of $E$. coli containing the zinc metalloprotease M60-like domain $\left(\mathrm{YghJ}_{\mathrm{M}}\right)$ that is involved in the pathogenesis of the enterotoxigenic strains via mucin degradation in the intestine.

Methods and Algorithms: To analyze amyloid properties of the $\mathrm{YghJ}_{\mathrm{M}}$ fibrils in vitro, transmission electron, confocal and polarization microscopies of the fibrils were used. For in vivo analysis, curli-dependent amyloid generator system (C-DAG), which provides export of the target protein to the surface of bacterial cells, was employed.

Results: We detected detergent-resistant aggregates of $\mathrm{YghJ}_{\mathrm{M}}$ by SDS-PAGE and SDDAGE and confirmed that these aggregates are resistant to $\alpha$-chymotrypsin protease treatment. We analyzed the fibrillary morphology of the obtained $\mathrm{YghJ}_{\mathrm{M}}$ aggregates using transmission electron microscopy. Next, we demonstrated that the $\mathrm{YghJ}_{\mathrm{M}}$ aggregates bind Thioflavin-T amyloid-specific dye and exhibit CD-spectra typical for protein aggregates rich in $\beta$-sheets. The Congo red stained $\mathrm{YghJ}_{\mathrm{M}}$ fibrils demonstrated apple-green birefringence which is considered to be the "gold standard" for verification of the amyloid structure. Finally, we showed that $\mathrm{YghJ}_{\mathrm{M}}$ forms amyloid fibrils on the surface of the Escherichia coli cells using C-DAG [3].

Conclusion: We demonstrated that $\mathrm{YghJ}_{\mathrm{M}}$ forms amyloid fibrils in vitro and in vivo. Our data on the amyloid properties of the YghJ protein highlight the role of this protein in the pathogenesis and suggest possible mechanism for its functioning.

Acknowledgements: Supported by the Grants of the President of the Russian Federation (MK-3240.2017.4, MK-512.2017.4) and by RFBR (16-34-60153, 17-54-150002).

\section{References}

1. Nizhnikov A.A. et al. (2016) Interaction of prions causes heritable traits in Saccharomyces cerevisiae. PLoS Genetics. 12(12). e1006504.

2. Antonets K.S. et al. (2016) Proteomic analysis of Escherichia coli protein fractions resistant to solubilization by ionic detergents. Biochemistry (Moscow). 81(1):34-46.

3. Belousov M.V. et al. (2018) M60-like metalloprotease domain of the Escherichia coli YghJ protein forms amyloid fibrils. PLoS ONE. 13(1):e0191317. 